\title{
EFEITO DO JEJUM NO INTERVALO ENTRE O NASCIMENTO E O ALOJAMENTO SOBRE O DESEMPENHO DE FRANGOS DE CORTE PROVENIENTES DE MATRIZES DE DIFERENTES IDADES
}

\section{(Effect of feed deprivation during the post hatch holding birth time on the performance of broiler chicks from breeders of different age)}

\author{
ALMEIDA, J.G. ${ }^{1}$ DAHLKE, F. ${ }^{2}$ MAIORKA, A. ${ }^{2}$ MACARI, M. ${ }^{3}$; FURLAN, R.L. ${ }^{3}$ \\ 'Pós-graduação em Zootecnia da Universidade Estadual Paulista/Faculdade de Ciências Agrárias e \\ Veterinárias/ Jaboticabal-SP, Brasil; \\ 2Universidade Federal do Paraná/Setor de Ciências Agrárias/Curitiba - PR, Brasil; \\ ${ }^{3}$ Universidade Estadual Paulista/Faculdade de Ciências Agrárias e Veterinárias/Jaboticabal-SP, Brasil.
}

\begin{abstract}
RESUMO - Este estudo foi conduzido para avaliar o efeito do jejum entre o nascimento e o alojamento sobre o consumo de ração, ganho de peso, conversão alimentar e viabilidade criatória até 42 dias de idade; perda de peso e consumo de ração no período que suceder ao nascimento, em frangos de corte provenientes de matrizes de diferentes idades. Foram utilizados 480 pintainhos de corte, machos, em um delineamento inteiramente casualizado, em esquema fatorial $2 \times 3$ com os fatores idades de matriz (30 e 60 semanas) e períodos de jejum (0, 24 e 48 horas póseclosão). A idade da matriz não influenciou a perda de peso dos pintainhos entre o nascimento e o alojamento, variável esta afetada apenas pelo período de jejum a qual as aves foram submetidas. Pintainhos oriundos de matizes de 60 semanas de idade consumiram maior quantidade de ração nas 72 horas pós-eclosão. A idade da matriz influenciou também o consumo de ração e o ganho de peso aos 7 e 21 dias de idade, sendo que pintainhos oriundos de matrizes mais velhas tiveram maior consumo e ganho de peso. No entanto, aos 42 dias, mesmo que as aves tenham sido submetidas a períodos de jejum pós-natal, não houve diferença no desempenho. Pintainhos oriundos de matrizes de 30 semanas tiveram melhor conversão alimentar nesta idade.
\end{abstract}

Palavras-chave: alojamento; frango de corte; idade de matriz; jejum pós-eclosão.

\footnotetext{
ABSTRACT - The present research work hás been carried out in order to evaluate on the post born period of broilers from different broiler breeders age, the effect of feed deprivation between hatching and housing, on feed intake, weight gain, feed conversion and viability up to 42 days old, and weight lost. A total of 480 male chicks were distributed in a randomized factorial design of $3 \times 3$, with two broiler breeders age ( 30 and 60 weeks) and three feed deprivation times $(0,24,48 \mathrm{~h}$ post hatching). The broiler breeder age did not affect the chicks lost of weight between hatching and housing, but the feed deprivation time affect this parameter.
}

Chicks from 60-week-old broiler breeders showed greater feed intake 72 hours post hatching. Feed intake and weight gains were influenced by the breeder age of 7 and 21 days old, the chick from older breeder siplaying greater feed intake and weight gain. However, at 42 days of age, even if the birds had been subjected to periods of post hatching fasting, they did not display differences on their performance. Chicks from 30-week-old broiler breeders displayed a better feed conversion.

Key-words: post hatch birth time; broiler breeder age; placement; broiler.

\section{Introdução}

No Brasil, quase três bilhões de pintainhos são transportados anualmente dos incubatórios para as unidades de criação. Diversos fatores podem determinar a duração do jejum pré-alojamento, como a variabilidade do número de horas até o rompimento da casca, temperatura de incubação, tamanho do ovo e época do ano. Isto faz com que dentro de uma mesma incubadora haja pintainhos com diferenças de tempo de eclosão de 36 a 48 horas (SKLAN et al., 2000; VIEIRA et al., 2005) Existem ainda os fatores operacionais do incubatório, como o tempo necessário para a sexagem e vacinação dos pintainhos. E por último, e não menos importante, tem o tempo de viagem. Todos estes fatores podem determinar perdas de peso do pintainho de até 10\% (CANÇADO e BAIÃO, 2002a, b).

Logo após a eclosão, a maior parte da demanda de energia e proteína das aves é direcionada para o desenvolvimento do trato digestório, principalmente intestinos (FISCHER DA SILVA, 2001). Este crescimento preferencial ocorre tanto na presença quanto na ausência do alimento (LAURENTIZ et al, 2001). Quando estes nutrientes não são fornecidos através da ração, os neonatos utilizam o saco vitelino como suplemento energético e como fonte protéica para o crescimento intestinal. Entretanto, DIBNER et al. 
Efeito do intervalo entre o nascimento e o alojamento sobre o desempenho de...

(1998) citam que $20 \%$ da proteína residual do saco vitelino são representadas pelas imunoglobulinas maternais, e que a gordura bruta residual é constituída basicamente de triglicérides, fosfolipídios e colesterol. É evidente que o uso destes nutrientes para fins nutricionais priva o neonato da proteção de anticorpos. Além disso, fosfolipídios e colesterol não são uma eficiente via de utilização da energia sendo mais eficientes quando utilizados como componentes essenciais das membranas celulares. Se todo o triacilglicerol presente no saco vitelino fosse metabolizado com $100 \%$ de eficiência, o máximo de energia oferecida seria de nove kcal, o que representa menos do requerimento energético para mantença do neonato no primeiro dia que é de $11 \mathrm{kcal}$. (DIBNER et al., 1998)

MAIORKA (2002) constatou que o trato digestório de pintainhos provenientes de matrizes velhas apresenta-se mais desenvolvido no momento da eclosão, fato que pode contribuir para uma melhor adaptação a alimentação exógena e também para um melhor desempenho destes animais (HUDSON et al., 2004).

O presente estudo tem por objetivo avaliar o efeito do período de jejum entre o nascimento e o alojamento sobre o desempenho de frangos de corte provenientes de matrizes de diferentes idades.

\section{Material e Métodos}

Foram utilizados 480 pintainhos, machos, de um dia de idade, da linhagem híbrida comercial Cobb-500®, sendo 240 pintainhos provenientes de matrizes de 30 semanas e 240 de reprodutoras com 60 semanas de idade. As aves eram oriundas de ovos produzidos em uma granja comercial, de matrizes de um mesmo lote, para cada uma das categorias (idade de matriz). O peso médio dos ovos, coletados no mesmo dia e horário, foi de $36 \mathrm{~g}$ e $45 \mathrm{~g}$ de matrizes de 30 e 60 semanas, respectivamente. Foram selecionados para o ensaio os pintainhos eclodidos com o tempo 496 horas de incubação.

As aves foram alojadas em diferentes intervalos: imediatamente após nascimento, 24 e 48 horas póseclosão. Foram distribuídas em boxes, recobertos com maravalha, equipados com bebedouros pendulares automáticos e comedouros tubulares, com manejo semelhante às criações comerciais. Os frangos receberam durante todo o período experimental (42 dias) luminosidade constante ( 24 horas) e alimento ad libitum. As rações, à base de milho e farelo de soja, foram formuladas segundo as exigências sugeridas pelo NRC (1994).

Para a análise das variáveis de desempenho (consumo de ração, ganho de peso e conversão alimentar) e perda de peso nas primeiras horas (24 e 48 horas), os animais foram pesados no momento do recebimento (chegada ao aviário) e no momento exato do alojamento, de acordo com os tratamentos propostos pelo ensaio. Também foram feitas pesagens dos animais e ração aos 7, 21 e 42 dias de idade.

$\mathrm{O}$ delineamento experimental foi inteiramente ao acaso, distribuído em um modelo fatorial $2 \times 3$, ( 2 idades de matriz: 30 e 60 semanas e períodos de jejum: 0, 24 e 48 horas pós eclosão), constituindo 6 tratamentos com 4 repetições por tratamento, sendo cada repetição composta de 20 aves. Os dados foram submetidos à análise de variância e para as características em que o valor da probabilidade se mostrou significativo $(P>0,05)$, foi aplicado o teste de Tukey, através do patoce estatístico SAS (2002).

\section{Resultados e Discussão}

Os pesos médios das aves no momento do alojamento e a perdas de peso decorrentes dos intervalos entre nascimento e alojamento são apresentados na TABELA 1. Pintainhos oriundos de matrizes mais velhas foram em média $9 \mathrm{~g}(\mathrm{p}<0,05)$ mais pesados do que aqueles oriundos de reprodutoras de 30 semanas de idade, no momento do alojamento. Animais alojados após períodos de jejum de 24 ou 48 horas foram mais leves do que os pintainhos alojados imediatamente após nascimento $(p<0,05)$. Da mesma forma, a ausência do alimento por períodos prolongados (24 ou 48 horas) resultou em proporcional perda de peso, quando expresso em valores absolutos, e também, da forma significativa quando expressa na forma percentual.

Reduções de peso de pintainhos também foram observadas por FISCHER DA SILVA (2001) que constataram perda de peso entre 3,5 e $6,5 \mathrm{~g}$, nas primeiras 48 horas de jejum das aves. A diminuição de peso é atribuída a uma maior utilização das reservas do saco vitelino, perdas das excreções digestiva e renal e pela desidratação, que são mais pronunciadas nesta fase (HALEVY et al., 2000). Esta redução, no entanto, não é influenciada pela idade da matriz $(p>0,05)$.

O consumo de ração nas primeiras horas após o alojamento (24, 48, 72 horas) é expresso na TABELA 1. Os pintainhos provenientes de matrizes mais velhas consumiram mais ração $(p<0,05)$ durante todo o período avaliado. Este comportamento ingestivo está diretamente relacionado ao tamanho do pintainho, que da mesma forma, é mais expressivo em animais provenientes de matrizes mais velhas. 
TABELA 1 - PESO (G) NO MOMENTO DO ALOJAMENTO (A), PERDA DE PESO (G) ENTRE O NASCIMENTO E ALOJAMENTO (B), PERCENTUAL DE PERDA DE PESO (C) E CONSUMO DE RAÇÃO (G) AS 24, 48 E 72 HORAS APÓS NASCIMENTO, DE PINTAINHOS PROVENIENTES DE MATRIZES DE DIFERENTES IDADES, COM DIFERENTES TEMPOS DE ALOJAMENTO.

\begin{tabular}{|c|c|c|c|c|c|c|c|}
\hline & \multicolumn{3}{|c|}{ Perda de Peso } & \multicolumn{4}{|c|}{ Consumo de ração } \\
\hline & $A *$ & $\mathrm{~B} *$ & $\mathrm{C} *$ & $24 \mathrm{~h}$ & $48 \mathrm{~h}$ & $72 \mathrm{~h}$ & Total \\
\hline \multicolumn{8}{|l|}{ Idade da Matriz } \\
\hline 30 Semanas & $36.46 \mathrm{~b}$ & 3.36 & 8.45 & $7 \mathrm{~b}$ & $10 \mathrm{~b}$ & $14 \mathrm{~b}$ & $23 \mathrm{~b}$ \\
\hline 60 Semanas & $45.53 \mathrm{a}$ & 4.02 & 8.17 & $8 \mathrm{a}$ & $14 \mathrm{a}$ & $16 \mathrm{a}$ & $27 \mathrm{a}$ \\
\hline \multicolumn{8}{|c|}{ Tempo Alojamento } \\
\hline $0 \mathrm{~h}$ & $44.19 \mathrm{a}$ & $0.80 \mathrm{a}$ & $1.74 \mathrm{a}$ & 8 & 11 & 16 & $35 \mathrm{a}$ \\
\hline $24 \mathrm{~h}$ & $39.95 \mathrm{~b}$ & $4.60 \mathrm{~b}$ & $10.30 \mathrm{~b}$ & - & 12 & 15 & $26 \mathrm{~b}$ \\
\hline $48 \mathrm{~h}$ & $38.84 \mathrm{~b}$ & $5.70 \mathrm{c}$ & $13.00 \mathrm{~b}$ & - & - & 13 & $15 \mathrm{c}$ \\
\hline \multicolumn{8}{|c|}{ Probabilidades } \\
\hline Idade & 0.001 & 0.078 & 0.743 & 0.011 & 0.007 & 0.025 & 0.001 \\
\hline Tempo & 0.001 & 0.001 & 0.001 & 0.020 & 0.869 & 0.081 & 0.001 \\
\hline Idade $\times$ Tempo & 0.385 & 0.840 & 0.516 & 0.025 & 0.368 & 0.375 & 0.828 \\
\hline C.V (\%) & 12.87 & 62.41 & 62.74 & - & - & 18.91 & 35.50 \\
\hline
\end{tabular}

Letras diferentes na coluna indicam diferença significativa $(p<0.05)$.

Quanto à quantidade total de alimento consumido no período inicial de 72 horas, verificou-se que aves não jejuadas apresentaram maior consumo do que àquelas submetidas a períodos de 24 ou 48 horas de jejum.

Pintainhos oriundos de matrizes mais velhas tiveram um maior consumo de ração aos 7, 21 e 42 dias, um maior ganho de peso aos 7 e 42 dias de idade (TABELA 2). Dados semelhantes foram observados por VIEIRA e MORAN (1998) e GONZALES et al. (2000). A diferença média no ganho de peso observada foi de $150 \mathrm{~g}$ para matrizes de 60 semanas aos 42 dias. Sendo que no primeiro dia pintainhos oriundos de matrizes de 60 semanas foram $9 \mathrm{~g}$ mais pesados, pode-se verificar que para cada $1 \mathrm{~g}$ de diferença no peso dos pintainhos no primeiro dia resultou em $17 \mathrm{~g}$ a mais no peso final.

Já aos 21 dias, foi observada interação significativa entre idade de matriz e tempo de alojamento para a variável ganho de peso (TABELA 2). Avaliando-se os intervalos de alojamento, isoladamente dentro de cada idade de matriz, percebe-se que o ganho de peso foi reduzido proporcionalmente à medida que aumentou 0 tempo para o alojamento das aves oriundas de matrizes de 30 semanas de idade. Esta característica é menos expressiva, no entanto, em pintainhos oriundos de matrizes de 60 semanas de idade, que não tiveram redução no ganho de peso, com período de jejum de 24 horas na sua fase inicial. Estes dados corroboram os resultados verificados por BAIÃO (1985) que não observou efeito de jejum de 24 horas sobre o ganho de peso e com MORAN (1990), PINCHASOV e NOY (1993), que observaram um pior desempenho de aves submetidas a um tempo de jejum de 48 horas.
Comparando-se os frangos provenientes de matrizes de diferentes idades, isoladamente em cada intervalo de alojamento, nota-se que independente do intervalo nascimento-alojamento, frangos provenientes de matrizes mais velhas tiveram maior ganho de peso até os 21 dias de idade.

Aves não alojadas logo após o nascimento tiveram menores consumo de ração aos 7, 21 e 42 dias de idade. Da mesma forma houve uma redução no ganho de peso aos 7 dias e no período total do experimento. O rápido acesso ao alimento após a eclosão pode trazer vantagens no desempenho das aves, uma vez que os nutrientes residuais do saco vitelino não são suficientes para suprir as necessidades do neonato, e, possivelmente, tenham maior valor quando aproveitados na forma de macromoléculas como imunoglobulinas e colesterol, do que na forma de aminoácidos e energia.

Esta diferença de consumo, juntamente com outros fatores fisiológicos, como produção enzimática, desenvolvimento de enterócitos nas criptas, aumento na quantidade e tamanho das vilosidades por área intestinal, favorecidas por um consumo precoce de alimento (MORAN, 1985), talvez possam explicar o melhor ganho de peso observado para aves não submetidas ao jejum. Essa maior quantidade de alimento ingerido por aves não jejuadas e provenientes de matrizes de idade avançada, provavelmente seja o principal fator que afeta o ganho de peso dos frangos na idade de abate (PINCHASOV e NOY, 1993).

A idade da matriz e o tempo de alojamento não afetaram a viabilidade criatória. 
Efeito do jejum no intervalo entre o nascimento e o alojamento sobre o desempenho de...

TABELA 2 - CONSUMO DE RAÇÃO (CR), GANHO DE PESO (GP) E CONVERSÃO ALIMENTAR (CA) DE FRANGOS DE CORTE, PROVENIENTE DE MATRIZES DE DIFERENTES IDADES COM DIFERENTES TEMPOS DE ALOJAMENTO.

\begin{tabular}{|c|c|c|c|c|c|c|c|c|c|c|c|c|}
\hline & \multicolumn{4}{|c|}{ Desempenho 1-7 dias } & \multicolumn{4}{|c|}{ Desempenho 1-21 dias } & \multicolumn{4}{|c|}{ Desempenho 1-42 dias } \\
\hline & $\mathrm{CR}$ & GP & $\mathrm{CA}$ & V.C & $\mathrm{CR}$ & GP & $\mathrm{CA}$ & V.C & $\mathrm{CR}$ & GP & $\mathrm{CA}$ & $\mathrm{VC}$ \\
\hline & $\mathrm{g}$ & $\mathrm{G}$ & $\mathrm{g} / \mathrm{g}$ & $\%$ & $\mathrm{~g}$ & $\mathrm{~g}$ & $\mathrm{~g} / \mathrm{g}$ & $\%$ & $\mathrm{~g}$ & $\mathrm{~g}$ & $\mathrm{~g}$ & $\%$ \\
\hline \multicolumn{13}{|l|}{ Idade da matriz } \\
\hline 30 semanas & $90 \mathrm{~b}$ & $74 \mathrm{~b}$ & 1,277 & 98.78 & $899 \mathrm{~b}$ & 621 & 1.487 & 97.53 & $3503 \mathrm{~b}$ & $2244 b$ & $1.699 \mathrm{a}$ & 95.31 \\
\hline 60 semanas & $116 a$ & $90 \mathrm{a}$ & 1,41 & 99.68 & $1081 a$ & 692 & $\begin{array}{c}1.516 \\
b\end{array}$ & 99.35 & 3929 a & $2394 \mathrm{a}$ & $1.749 \mathrm{~b}$ & 96.15 \\
\hline \multicolumn{13}{|c|}{ Tempo alojamento } \\
\hline 0 & $131 \mathrm{a}$ & $99 a$ & 1,409 & 99.04 & $1083 a$ & 700 & 1.574 & 98.56 & $3859 a$ & $2347 a$ & 1.746 & 93.27 \\
\hline 24 & $93 \mathrm{~b}$ & $84 \mathrm{~b}$ & 1,182 & 99.52 & $968 \mathrm{~b}$ & 664 & 1.553 & 98.56 & $3706 \mathrm{~b}$ & $2346 \mathrm{a}$ & 1.720 & 97.11 \\
\hline 48 & $86 \mathrm{~b}$ & $63 c$ & 1,44 & 99.14 & $919 c$ & 606 & 1.500 & 98.23 & $3582 \mathrm{c}$ & $2264 b$ & 1.706 & 96.81 \\
\hline & \multicolumn{12}{|c|}{ Probabilidades } \\
\hline Idade & 0,007 & 0 & 0,26 & 0.277 & 0.001 & 0.001 & 0.015 & 0.111 & 0.001 & 0.001 & 0.001 & 0.721 \\
\hline Tempo & 0,001 & 0 & 0,161 & 0.875 & 0.001 & 0.001 & 0.323 & 0.956 & 0.011 & 0.021 & 0.140 & 0.349 \\
\hline Idade x Tempo & 0,487 & 0,55 & 0,477 & 0.683 & 0.957 & 0.003 & 0.587 & 0.768 & 0.937 & 0.217 & 0.461 & 0.352 \\
\hline CV (\%) & 30.45 & 21.67 & 21.72 & 1.85 & 13.03 & 8.76 & 7.10 & 2.62 & 7.69 & 4.50 & 2.73 & 5.92 \\
\hline
\end{tabular}

Letras diferentes na coluna indicam diferença significativa $(p<0.05)$.

\section{Conclusões}

Nas condições em que foi conduzido o experimento, podemos concluir que a perda de peso de pintainhos neonatos não é influenciada pela idade das matrizes que os geraram.

Aves provenientes de matrizes mais velhas possuem maior ganho de peso e consumo de ração à idade de abate.

\section{Referências}

BAIÃO, N.C. Efeito do intervalo entre o nascimento e o alojamento de pintos sobre o desempenho de frangos de corte. In: CONGRESSO BRASILEIRO DE AVICULTURA, 1985. Anais... Brasília: CBA, 1985, p.3132.

CANÇADO, S.V.; BAIÃO, N.C. Efeito do período de jejum entre o nascimento e alojamento e da adição de óleo à ração sobre o desempenho de pintos de corte e digestibilidade da ração. Arquivos Brasileiros de Medicina Veterinária e Zootecnia, v.54, n.6, p.630-635, 2002.

CANÇADO, S.V.; BAIÃO, N.C. Efeito do período de jejum entre o nascimento e alojamento e da adição de óleo à ração sobre o desenvolvimento do trato gastrointestinal e atividade de lipase. Arquivos Brasileiros de Medicina Veterinária e Zootecnia, v.54, n.6, p. 623-629, 2002.

DIBNER, J.J.; KNIGHT, C.D.; KITCHELL, M.L.; ATWELL, C.A.; DOWNS, A.C.; IVEY, F.J. Early feeding and development of the immune system in neonatal poultry. Journal of Applied Poultry Research, v.7, 425-436, 1998.
FISHER DA SILVA, A.V. Efeitos da restrição alimentar precoce e da glutamina no desempenho e na mucosa intestinal em frangos. 2001.77 p.Tese (Doutorado em Zootecnia) - Faculdade de Ciências Agrárias e Veterinárias, Universidade Estadual Paulista.

GONZALES, E.; LEANDRO, N.S.M.; VAROLI JR, J.C.; TAKITA, T.S.; LODDI, M.M. Tempo jejum do neonato e a restrição alimentar precoce influenciando a produtividade de frangos de corte na idade de abate. Revista Brasileira de Ciência Avícola, Supl.2, p.4, 2000.

HALEVY, O.; GEYRA, A.; BARAK, M.; UNI, Z.; SKLAN, $D$. Early posthatch starvation decreases satellite cell proliferation and skeletal muscle growth in chicks. Journal of Nutrition, v.130, p.858-864, 2000.

HUDSON, B.P.; DOZIER, W.A.; WILSON, J.L.; SANDER, J.E.; WARD, T.L. Reproductive performance and immune status of caged broiler breeder hens provided diets supplemented with either inorganic or organic sources of zinc from hatching to 65 wk of age. Journal of Applied Poultry Research, Mississippi, v.13, p.349-359, 2004.

LAURENTIZ, A.C.; ARIKI, J.; ARAÚJO, L.F.; BORGES, S.A.; ARAÚJO, C.S.S.; MAIORKA, A. Adaptações digestivas pós-eclosão. In: CONFERÊNCIA APINCO DE CIÊNCIA E TECNOLOGIA AVÍCOLAS, 2001, Campinas. Anais. Campinas: FACTA, 2001. p.141-151.

MAIORKA, A. Efeito da idade da matriz, do jejum, da energia da ração e da glutamina sobre o desenvolvimento da mucosa intestinal e atividade enzimática do pâncreas de pintos de corte. 2002. 103f. Tese (Doutorado em Zootecnia) - Faculdade de Ciências Agrárias e Veterinárias, Universidade Estadual Paulista.

MORAN Jr, E.T. Digestion and absorption of carbohydrates in fowl through perinatal development. Journal of Nutrition, v.115, p.665-674, 1985. 
MORAN Jr, E.T. Effect of egg weight, glucose administration at hatch, and delayed access to feed and water on the poult at 2 weeks of age. Poultry Science, v.69, p.1718-1723, 1990.

NATIONAL RESEARCH COUNCIL, Nutrient Requeriments of Poultry. Washington D.C., $9^{\text {th }}$ Revised Edition National Research Council, National Academic, 1994. p.22-24.

PINCHASOV, Y.; NOY, Y. Comparison of post-hatch holding time and subsequent early performance of broiler chicks and turkey poults. British Poultry Science, v.34, p.110-120, 1993.

SAS, 2002. SAS User's Guide: Statistics. SAS Institute Inc., Cary, NY.
SKLAN, D.; NOY, Y.; HOYZMAN, A.; ROZENBOIM, I. Decreasing weight loss in the hatchery by feeding chicks and poults in hatching trays. Journal of Applied Poultry Research, v.9, p.142148, 2000.

VIEIRA, S. L.; MORAN, E.T. Broiler yields using chicks from extremes in breeder age and response to dietary propionate at placement. Journal Applied Poultry Research, v.7, p.320-327, 1998.

VIEIRA, S.L.; ALMEIDA, J.G.; LIMA, A.R.; CONDE, O.R.A.; OLMOS, A.R. Hatching distribution of eggs varying in weight and breeder age. Revista Brasileira de Ciência Avícola, v.7, n.2, p.73-78, 2005.

Recebido para publicação:

$21 / 06 / 2006$

Aprovado:

$10 / 09 / 2006$ 